\title{
Severe Itchy Extensive Psoriasis Vulgaris Provoked by Tramadol
}

\author{
Mohammed Abu El-Hamd ${ }^{1,{ }^{*}}$ and Soha Aboeldahab ${ }^{1}$ \\ ${ }^{1}$ Faculty of Medicine, Sohag University, Sohag, Egypt \\ "Corresponding author: Faculty of Medicine, Sohag University, Sohag, Egypt. Email: mohammedadva@yahoo.com \\ Received 2022 January 15; Revised 2022 February 01; Accepted 2022 February 01.
}

\begin{abstract}
To the best of our knowledge, we are reporting the first case of psoriasis vulgaris in which the lesions became extensive with marked itching and resistance to traditional lines of treatment after the use of tramadol. Thus, tramadol may be a new drug exacerbating psoriasis vulgaris that should be avoided. Tramadol increases the severity of psoriasis vulgaris and induces marked itching in these patients.
\end{abstract}

Keywords: Psoriasis Vulgaris, Tramadol, Skin Diseases

\section{Introduction}

Psoriasis is a chronic, immune-mediated inflammatory cutaneous disease associated with high physical and psychosocial burdens (1). The estimated prevalence of psoriasis is $11 \%$ in adults and $1.3 \%$ in children. Therefore, psoriasis is frequently encountered in clinical practice (2).

\section{Case Presentation}

A 34-year-old male worker, a heavy cigarette smoker, was treated 10 years ago for mild controlled psoriasis vulgaris with topical medications (corticosteroids and emollients). He presented with severe itchy extensive psoriatic lesions involving most of his body in the past three months, not responding to previous lines of treatment and interfering with his daily activities. There was no family history of psoriasis and no history suggestive of systemic diseases. The patient used tramadol three months ago to treat premature ejaculation by self-description and gradually increased the dose to gain the maximum response. Since then, psoriasis became gradually extensive with marked itching and resistance to usual lines of treatment. Surprisingly, the withdrawal of tramadol improved itching and stopped the progress of psoriasis lesions. Diagnosis of psoriasis was confirmed clinically and dermoscopically. Clinical cutaneous examination revealed severe excoriated disseminated psoriasis plaques. General examinations of the patient were normal (Figure 1).

\section{Discussion}

The etiology of psoriasis is multifactorial. A complex interplay between genetic and environmental factors leads to the onset of psoriasis. In addition, several exacerbating factors could trigger psoriasis, such as infection, physical trauma, and emotional stress $(3,4)$.

There are numerous drugs eliciting psoriasis in several ways. First, the drug can aggravate the preexisting psoriatic skin lesions (drug-aggravated psoriasis). Second, the drug can initiate new psoriasis lesions in patients with a personal history of psoriasis. Third, the drug can provoke psoriasis de novo in patients without personal or family history of psoriasis. Several drugs have been linked strongly to psoriasis, such as beta-blockers, antimalarial drugs, lithium, imiquimod, interferons, and terbinafine (5). Rapid withdrawal of systemic corticosteroids or potent topical corticosteroids may cause a psoriasis flare (6).

Diagnosis of drug-related exacerbation and initiation of psoriasis can be difficult in daily clinical practice for several reasons. First, the association between drugs and psoriasis onset and/or exacerbation is usually not wellevaluated, resulting in a low level of evidence strength. Second, the lag time between the start of medication and psoriasis onset varies and can be pretty lengthy. Third, the psoriasis flare can persist in some patients, even after the implicated drug is withdrawn. Therefore, the clinical diagnosis of drug-related psoriasis may be challenging, and recognizing potential drugs involved in psoriasis is of clinical importance to allow optimal management (1).

To the best of our knowledge, we reported the first case of psoriasis vulgaris in which the lesions became extensive 

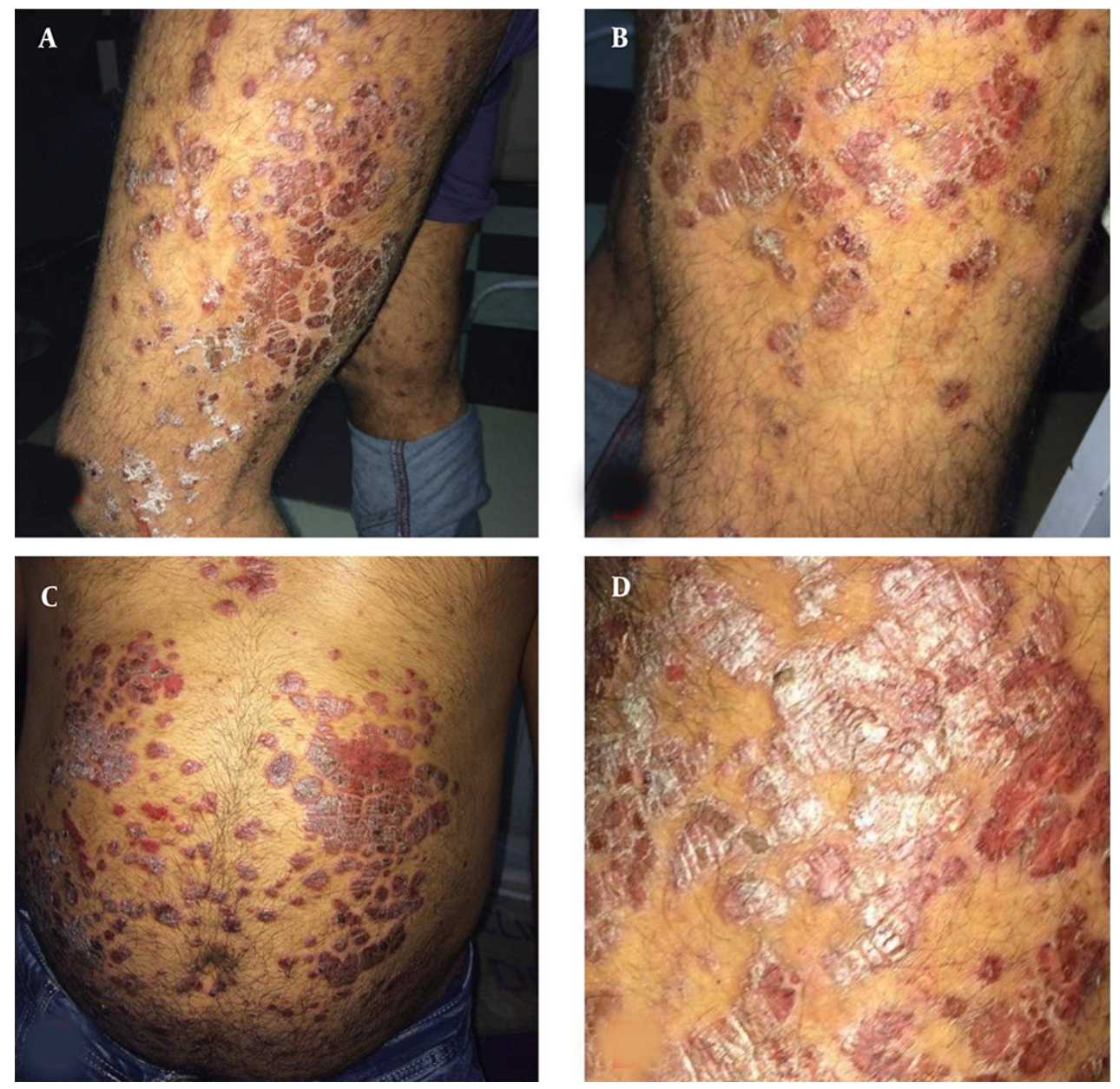

Figure 1. A34-year-old male patient presented with severe excoriated disseminated psoriasis vulgaris. (A) left thigh,(B) right thigh,(C)Abdomen, and(D) close view of psoriatic plaques.

with marked itching and resistance to traditional lines of treatment after the use of tramadol. Thus, tramadol may be a new drug exacerbating psoriasis vulgaris that should be avoided. Tramadol increases the severity of psoriasis vulgaris and induces marked itching in these patients.

\section{Footnotes}

Authors' Contribution: Study concept and design: M. A. and S. A.; Analysis and interpretation of data: M.A. and S. A.; Drafting of the manuscript: M. A. and S. A.; Critical revision of the manuscript for important intellectual content: F. M, A. and S. A.; Paper submission: M. A.

Conflict of Interests: All the authors declare no conflict of interest regarding this manuscript.

Funding/Support: All the authors declared no financial support regarding this manuscript.
Informed Consent: It was not declared by the authors.

\section{References}

1. Boehncke W, Schön MP. Psoriasis. Lancet. 2015;386(9997):983-94. doi: 10.1016/s0140-6736(14)61909-7.

2. Michalek IM, Loring B, John SM. A systematic review of worldwide epidemiology of psoriasis. J Eur Acad Dermatol Venereol. 2017;31(2):205-12. doi: 10.1111/jdv.13854. [PubMed: 27573025].

3. Lowes MA, Suarez-Farinas M, Krueger JG. Immunology of psoriasis. Annu Rev Immunol. 2014;32:227-55. doi: 10.1146/annurev-immunol032713-120225. [PubMed: 24655295]. [PubMed Central: PMC4229247].

4. Dika E, Bardazzi F, Balestri R, Maibach HI. Environmental factors and psoriasis. Curr Probl Dermatol. 2007;35:118-35. doi: 10.1159/000106419. [PubMed: 17641494].

5. Kim GK, Del Rosso JQ. Drug-provoked psoriasis: is it drug induced or drug aggravated?: understanding pathophysiology and clinical relevance. J Clin Aesthet Dermatol. 2010;3(1):32. 
6. Mrowietz U, Domm S. Systemic steroids in the treatment of psoriasis: what is fact, what is fiction? J Eur Acad Dermatol Venereol.
2013;27(8):1022-5. doi: 10.1111/j.1468-3083.2012.04656.x. [PubMed: 22830601]. 